\title{
Thoracic splenosis
}

\author{
Shahar Madjar, Dov Weissberg
}

\begin{abstract}
Background - Thoracic splenosis is an uncommon side effect of thoracoabdominal trauma involving injury to the diaphragm and spleen. Only 20 patients with a similar problem have been reported previously. Methods - Two patients with thoracic splenosis were studied.

Results - One of the patients had undergone thoracotomy for resection of a "pulmonary nodule". The other remains under observation.

Conclusions - Presence of a pulmonary nodule in a patient with history of injury to the diaphragm and spleen should arouse suspicion of splenosis. Appropriate investigation may prevent an unnecessary and potentially harmful operation.
\end{abstract}

(Thorax 1994;49:1020-1022)

Splenosis is defined as the autotransplantation of splenic tissue, usually after splenic rupture. It occurs usually in the peritoneum, omentum, and the mesentery, where nodules of splenic tissue may be found either at necropsy or at subsequent laparotomy. Thoracic splenosis usually follows a traumatic event involving the spleen and the diaphragm. This is an uncommon phenomenon with only 20 instances being published in the English literature..$^{1-3}$ We report two additional patients with this complication and review the literature.

\section{Patient 1}

At the age of 18 this patient had attempted suicide by shooting himself in the chest. The entrance wound was near the left border of the sternum and the exit wound in the back near the base of the left lung. Thoracotomy disclosed injury to the lingula, the pericardium, and the diaphragm. The abdominal organs were explored through an incision in the diaphragm, revealing extensive rupture of the spleen, a small tear of the left lobe of the liver, and a laceration of the lesser curvature of the stomach. The spleen was resected and the liver, stomach, and diaphragm were sutured. In 1985, at the age of 47, he underwent a Billroth II gastrectomy for a bleeding gastric ulcer. At laparotomy several nodules were found within the abdominal cavity which were resected and reported to be splenic tissue. A chest radiograph taken during this admission (fig 1) was reported

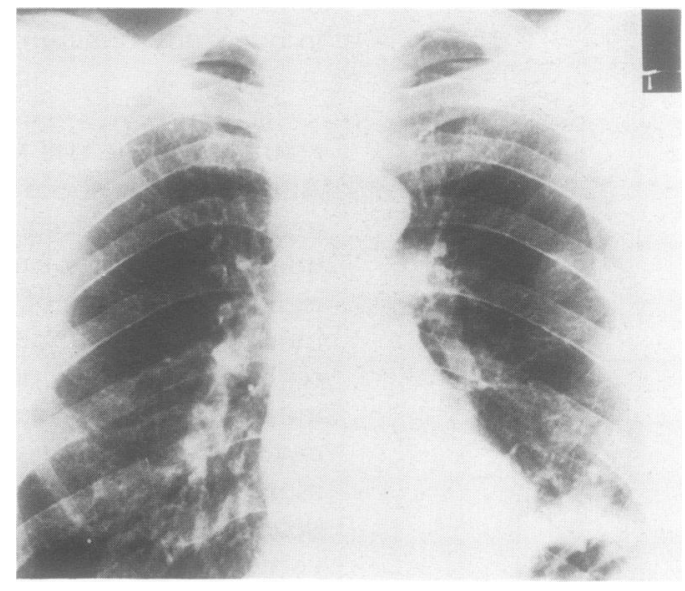

Figure 1 Chest radiograph interpreted as normal. The nodule in the left mid lung field was missed.

as normal (on review there is a small nodule in the left mid zone). In 1987 he was admitted to the chest unit following a routine chest radiograph (fig 2 ) which showed a $3 \mathrm{~cm}$ mass in the left mid zone. This was resected with a wedge of pulmonary tissue and histological examination confirmed the lesion as splenic tissue. A chest radiograph taken in 1990 has shown a further pulmonary nodule near the apex of the left lung. On hindsight this lesion was present on the chest radiograph taken in 1987 (fig 2). This nodule will be managed conservatively.

\section{Patient 2}

This 40 year old man was involved at the age of 10 years in a street brawl with his classmates

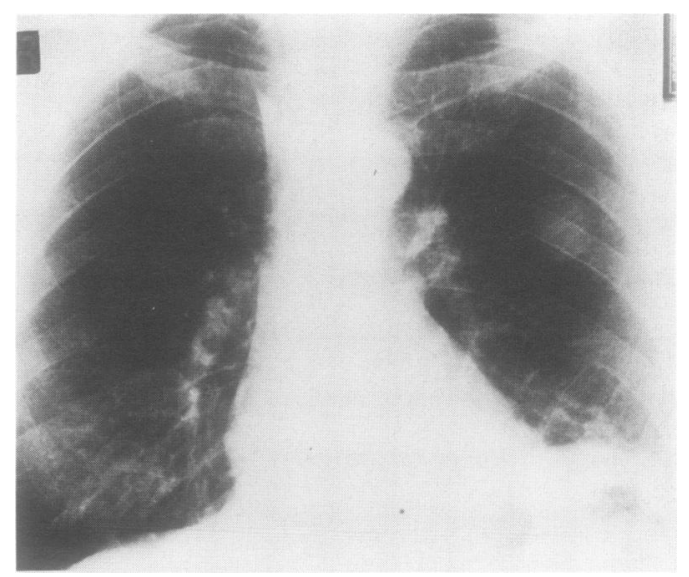

Figure 2 The nodule not noted two years earlier has increased in size. Another density behind the left clavicle did not rouse suspicion. 


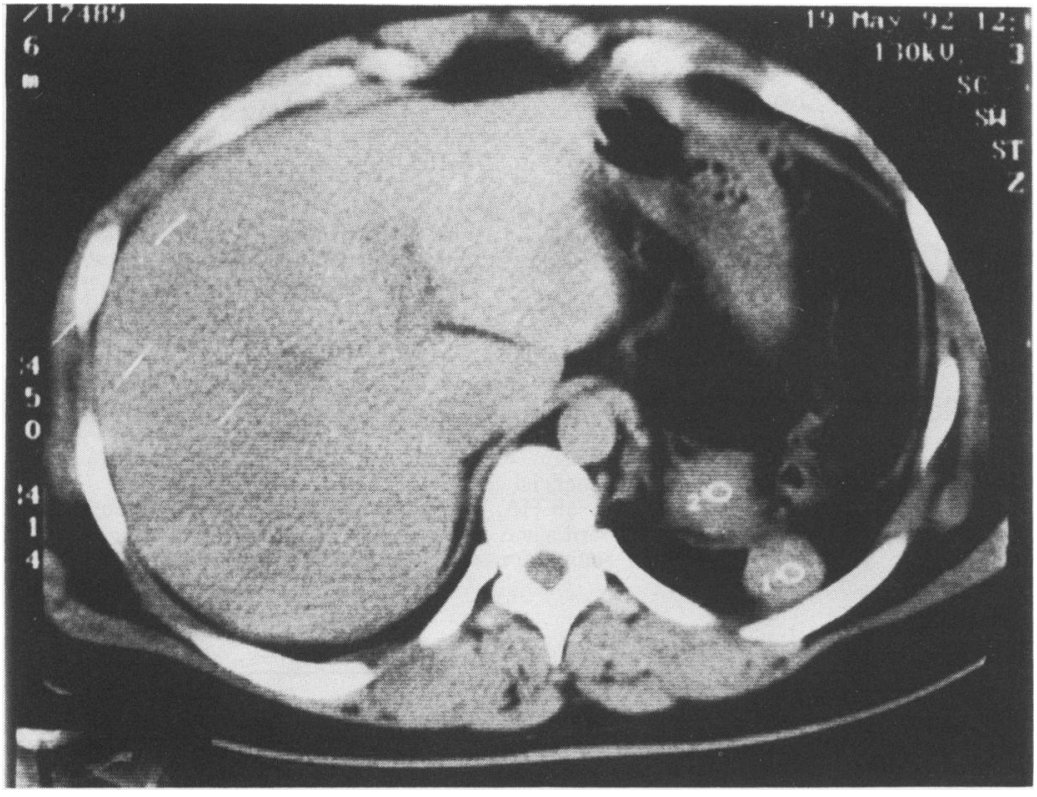

Figure 3 Computed tomographic scan of patient 2 showing two masses in close proximity to the diaphragm, above and below.

in Russia, which ended with the patient being thrown under the wheels of a passing truck. Findings at his operation included lacerations of the spleen, stomach, diaphragm, and left lung. A splenectomy, partial gastrectomy, and left lower lobectomy were performed. At the age of 38 he immigrated to Israel and a routine radiograph showed a mass in the mediastinum. A computed tomographic scan confirmed that there were two nodules in the left hemithorax located posteriorly close to the diaphragm, an abdominal mass close to the diaphragm, and a mediastinal mass (fig 3). ${ }^{99 m}$ Technetiumlabelled sulphur colloid scanning suggested that all the masses represented splenic tissue. No active treatment was indicated and the patient is well and works as a physician.

\section{Discussion}

In 1937 Shaw and Shafi described the first case of post-traumatic intrathoracic splenosis discovered at necropsy. ${ }^{1}$ The cause of death was not related to the lesion which had been asymptomatic during life. During the following years 19 additional cases of thoracic splenosis have been described in the English medical literature. ${ }^{23}$ There were 15 men and five women, ranging in age from 15 to 59 years. All patients had a history of combined abdominal and thoracic trauma. The causative agent was a gunshot wound in 11 instances, shrapnel injury in two, motor vehicle accident in four, and unknown circumstances in three. Splenectomy was performed in all patients, together with suturing the diaphragm in 10 , and pulmonary resection in one. The thoracic splenosis became evident 6-42 years after the injury. The nodules were multiple in 10 patients and solitary in 10. All lesions were pleurally based, and all were in the left hemithorax. At the time of diagnosis all the patients were asymptomatic with the exception of one individual who had recurrent episodes of haem- optysis. This is in contrast to abdominal splenosis in which abdominal pain, intestinal obstruction, gastrointestinal bleeding, and the recurrence of haematological disorders have been described. Diagnostic investigations have included computed tomography, radionuclide scanning, needle aspirations, and needle biopsies. The findings on computed tomography showed characteristic pleurally based pulmonary nodules, but were not diagnostically specific. Both needle biopsies and two of the six fine needle aspirations were diagnostic. The other four needle aspirations produced only lymphoid tissue and red blood cells. Radionuclide scanning was used in six patients. The ${ }^{99 \mathrm{~m}}$ technetium-labelled sulphur colloid scanning showed increased uptake in all instances, correlating well with the radiographic location of the mass. Scanning with ${ }^{11}$ indium-labelled platelets and ${ }^{99 \mathrm{~m}}$ Tc-labelled heat damaged red cells was tried successfully in two patients. On four occasions the diagnosis was established on radionuclide scanning alone. ${ }^{4-7}$ In one instance the diagnosis was made at necropsy, ${ }^{1}$ and in 11 patients at operation, when reddish-brown pleurally based nodules measuring from a few $\mathrm{mm}$ to $7.5 \mathrm{~cm}$ were observed. Based on current experience we believe that, of all the tests available, the heat damaged red cell scan is the most specific for splenic tissue and should be recommended as the method of choice in establishing the diagnosis.

The sequence of events in our first patient deserves some further comment. The chest radiograph taken during the patient's admission for gastrectomy in 1985 (fig 1) revealed a left mid-zone pulmonary nodule, albeit small and inconspicuous. At the time it had been overlooked. Apparently it had been present there for three decades and had not changed until resection of the abdominal speniculi. This stimulated its growth over the subsequent two years, so that in 1987 it had reached $3 \mathrm{~cm}$ in diameter and was resected as a suspicious pulmonary nodule. A new pulmonary nodule near the apex seen on a radiograph in 1990 may be an apparent expression of yet another such stimulus brought about by the resection of the first pulmonary nodule in 1987.

There is an increasing awareness of potentially lethal sepsis following splenectomy. At least 42 instances of overwhelming postsplenectomy infection have been reported. ${ }^{8}$ The longest interval between extirpation of the spleen and subsequent sepsis was 42 years, indicating a small lifelong risk of severe infection in asplenic patients. Accordingly, resection of splenic tissue should be avoided when splenosis is diagnosed either before or during the operation. If during operation the entire spleen cannot be saved, at least some splenic tissue should be retained and implanted in the omentum. ${ }^{910}$ However, all patients after total splenectomy should receive Pneumovax and Haemophilus influenzae vaccines. In addition, children should be treated with oral penicillin daily until the age of 18 .

Individuals with a history of thoracoabdominal trauma with ruptured spleen are at risk of developing thoracic splenosis. Most of 
these patients remain asymptomatic, and left sided pleural or pulmonary nodules may be discovered many years after the episode of trauma. Pulmonary nodules are often malignant, and it is commonly accepted that they should be considered malignant unless proven otherwise. Alertness to the possibility of thoracic splenosis can lead to confirmation of the diagnosis by the use of radionuclide scanning, and so prevent unnecessary thoracotomy and rendering the patient asplenic.

1 Shaw AFB, Shafi A. Traumatic autoplastic transplantation of splenic tissue in man with observations on the late results of splenectomy in six cases. 7 Pathol 1937;45. 215-35.

2 Roucos S, Tabet G, Jebara VA, Ghossain MA, Biagini J,
Saade B. Thoracic splenosis: case report and literature review. I Thorac Cardiovasc Surg 1990;99:361-3.

3 Hietala E-M, Hermunen H, Kostiainen S. Intrathoracic splenosis: report of a case simulating esophageal leiomyoma. Scand f Thorac Cardiovasc Surg 1993;27:61-3.

4 Scales FE, Lee ME. Nonoperative diagnosis of intrathoracic splenosis. AfR 1983;141:1273-4.

5 Moncada R, Williams V, Fareed J, Messmore H. Thoracic splenosis. $A F R$ 1985;144:705-6.

6 Schiff RG, Leonidas J, Schende A, Lanzkowski P. The noninvasive diagnosis of intrathoracic splenosis using technetium- $99 \mathrm{~m}$ heat-damaged red blood cells. Clin Nucl Med 1987;12:785-7.

7 Hart CM, Clore FC, Hood CI. Intrathoracic splenosis. Chest 1990;97:1024.

8 Sass W, Bergholz M, Kehl A, Seifert J, Hamelmann H. Overwhelming infection after splenectomy in spite of some spleen remaining. Klin Wochenschr 1983;61:1075-9.

9 Widmann WD, Laubscher FA. Splenosis: a disease or a beneficial condition? Arch Surg 1971;102:152-8.

10 Pearson HA, Johnston D, Smith KA, Touloukian RJ. The born again spleen: return of splenic function after splenectomy for trauma. N Engl ₹ Med 1978;298:1389-92.

\title{
Effects of nedocromil sodium in the treatment of non-allergic subjects with chronic obstructive pulmonary disease
}

\author{
Jan W de Jong, Dirkje S Postma, Thomas W van der Mark, Gerard H Koëter
}

Department of Pulmonology, University Hospital, 9713 EZ Groningen, The Netherlands $\mathrm{J}$ W de Jong D S Postma $T$ W van der Mark G H Koëter

Reprint requests to: Professor D S Postma.

Received 8 November 1993 Returned to authors 7 January 1994 Revised version received 10 March 1994 Accepted for publication 27 May 1994

\begin{abstract}
Background - Nedocromil sodium, a nonsteroidal anti-inflammatory drug, is effective in the treatment of asthma. Its efficacy in the treatment of chronic obstructive pulmonary disease (COPD) has not been investigated.

Methods - Fifty four non-allergic patients with COPD were randomised to 10 weeks of treatment with placebo or nedocromil sodium $(4 \times 8 \mathrm{mg} /$ day) in a double blind study.

Results - Nedocromil sodium treatment had no effect on airway responsiveness to histamine, methacholine, and adenosine5'-monophosphate, pulmonary function, and symptom scores. Both patients and clinicians favoured treatment with nedocromil sodium, however, and the number of dropouts (because of exacerbations) was fewer during treatment with the drug. Conclusions - Longer trials will be necessary to assess if nedocromil sodium can reduce the frequency of exacerbations and the decrease in pulmonary function, eventually leading to a better quality of life in patients with COPD.
\end{abstract}

(Thorax 1994;49:1022-1024)

Nedocromil sodium has been shown to be effective in the treatment of patients with al- lergic asthma. ${ }^{1}$ Studies investigating its effects in chronic obstructive pulmonary disease (COPD) are not available. The aim of our study was to investigate the effects of 10 weeks of treatment with nedocromil sodium on airway responsiveness to histamine, methacholine, and adenosine- $5^{\prime}$-monophosphate (AMP), pulmonary function, and clinical symptoms in a group of non-allergic patients with COPD. As anti-inflammatory treatment in COPD is generally accompanied by little or no change in clinical parameters a high dose of nedocromil sodium (arbitrarily determined at $32 \mathrm{mg}$ daily) was chosen.

\section{Methods}

SUBJECTS

All participants were at least 40 years of age and had a diagnosis of COPD based on the recommendations of the American Thoracic Society, ${ }^{2}$ meeting the following criteria: (1) no atopy: negative skin reactions to 18 common aeroallergens, negative specific serum immunoglobulin $\mathrm{E}$ to house dust mite, and peripheral blood eosinophils $<450 / \mathrm{mm}^{3}$; (2) forced expiratory volume in one second $\left(\mathrm{FEV}_{1}\right)>1$ litre and $<70 \%$ predicted and $\mathrm{FEV}_{1}$ after $40 \mu \mathrm{g}$ ipratropium bromide $<80 \%$ predicted; (3) concentration of histamine causing a $20 \%$ decrease 